\title{
Uso, omisión e interpretación del artículo definido en hispanohablantes de herencia*
}

\author{
Use, omission and interpretation of the definite article in heritage Spanish \\ speakers
}

\author{
Rosalva Alamillo \\ University of Houston, Department of Hispanic Studies
}

\section{Resumen}

¿Por qué los hablantes de español como lengua de herencia presentan variabilidad en el uso del artículo definido (ArtD) en español? Se ha establecido que las construcciones nominales definidas (Las mesas grandes) y escuetas (Mesas grandes) tienen diferente interpretación y distribución en inglés y español (Chierchia, 1998; Gelman \& Raman, 2003; Dayal, 2004; Montrul \& Ionin, 2010, 2012; entre otros). El objetivo es investigar la transferencia de los aspectos sintáctico (uso vs omisión del ArtD) y semántico (interpretación gené- rica vs interpretación específica) en sintagmas nominales en la escritura de hispanohablantes de herencia. Se analizaron ciento sesenta y cuatro composiciones que presentaban nombres plurales contables y singulares no contables. Se encontró transferencia del inglés al español en cuatro niveles de competencia lingüística, pero esta disminuye conforme avanza el dominio de la lengua de herencia. Finalmente, se discute cuál de las dos propiedades es más susceptible a superar las diferencias entre las lenguas.

Palabras clave: nombres definidos; nombres escuetos; transferencia semántica; transferencia sintáctica; español como lengua de herencia

Agradezco sinceramente a mi directora de tesis doctoral, la Dra. Marta Fairclough, por las invaluables enseñanzas que me ha transmitido en el área del español como lengua de herencia, así como por su constante e incondicional apoyo. También agradezco a los dos dictaminadores anónimos que revisaron el manuscrito y contribuyeron a la mejora de este artículo. 


\begin{abstract}
Why do heritage Spanish speakers exhibit tion vs generic interpretation) in nominal variability in the use of the Spanish defi- phrases in the writing of heritage Spanish nite article? Previous studies have shown speakers. One hundred and sixty-six texts that definite nouns (Las mesas grandes) including count plurals and mass singulars and bare nouns (Mesas grandes) differ in were analyzed. Transfer from English into their interpretation and distribution in both Spanish was observed at all four language English and Spanish (Chierchia, 1998; proficiency levels, although it decreases Gelman \& Raman, 2003; Dayal, 2004; as proficiency in the heritage language inMontrul \& Ionin, 2010, 2012; among oth- creases. Finally, the susceptibility of these ers). This study aims to examine the trans- two properties (syntactic and semantic) to fer of syntactic (definite vs. bare nous) cross-linguistic transfer is also discussed. and semantic aspects (specific interpreta-
\end{abstract}

Keywords: definite nouns; bare nouns; semantic transfer; syntactic transfer; Spanish as a heritage language 


\section{Introducción}

Este estudio investiga la transferencia sintáctico-semántica en el uso de plurales contables y singulares no contables en hablantes de español como lengua de herencia, cuya lengua dominante es el inglés. Desde el punto de vista pedagógico, es necesario clarificar qué aspectos estructurales y semánticos son problemáticos para los hablantes de español como lengua de herencia con el fin de ofrecer una mejor instrucción en las aulas y desarrollar materiales educativos adecuados — según los elementos lingüísticos que interfieren de un sistema a otro en los hablantes bilingües. Los participantes de esta investigación son candidatos al programa de español como lengua de herencia de una universidad urbana en Texas, Estados Unidos. Fueron divididos en grupos según la competencia lingüística que mostraron tener en el examen de ubicación para entrar a dicho programa.

Texas cuenta con una comunidad hispana bilingüe que muestra una preferencia de uso del inglés en detrimento del uso del español conforme avanzan las generaciones de inmigrantes hispanos. Típicamente esto ocurre porque los hablantes han tenido una mayor exposición al inglés, en especial en lo referente a la escolarización. El tener una mayor competencia lingüística en una lengua -en este caso el inglés - provoca que los hablantes se sientan más cómodos usando dicha lengua y esta se convierta en la lengua de uso preferida (Austin, Blume \& Sánchez, 2015: 57). En la población hispana de Texas, la preferencia de uso del inglés y la falta de educación formal en español están intrínsecamente relacionadas con las políticas educativas estadounidenses que, hoy por hoy, no muestran interés en la conservación de las lenguas de herencia.

Es importante mencionar que el término lengua de herencia se refiere, en la mayoría de los casos, a una lengua minoritaria que cohabita un mismo contexto con una lengua mayoritaria. La diferenciación entre lenguas minoritarias y ma-

yoritarias es esencialmente sociopolítica. La idea de que la lengua mayoritaria es más prestigiosa también contribuye al desuso o abandono paulatino de la lengua de herencia.

Asimismo, el término lengua de herencia hace referencia a una lengua que se adquirió a temprana edad, pero que en la vida adolescente o adulta no es necesariamente la lengua dominante del hablante (Benmamoun, Montrul \& Po- 
linsky, 2013: 2-3). En los hablantes de lengua de herencia existen diferentes niveles de competencia lingüística: desde aquellos que solo posen habilidades receptivas (entendimiento de la lengua) hasta los que se comunican efectivamente en la lengua de herencia de forma oral y escrita. En cuanto a los participantes de este estudio, la definición que propone Guadalupe Valdés los perfila de forma más específica:

the term "heritage speaker" is used to refer to a student of language raised in a home where a non-English language is spoken, who speaks or merely understands the heritage language, and who is to some degree bilingual in English and the heritage language [el término hablante de herencia se usa para referirse a un estudiante de lengua que ha sido criado en un hogar donde se habla una lengua distinta al inglés, quien habla o solo entiende la lengua de herencia y es, en cierto grado, bilingüe en inglés y la lengua de herencia] (Valdés, 2000: 375; la traducción es mía).

Generalmente, la lengua dominante de estos hablantes es el inglés, por lo que la lengua de herencia suele presentar ciertos vacíos en distintos niveles lingüísticos; de ahí la referencia a "cierto grado" de bilingüismo.

El objetivo de esta investigación es determinar si hay transferencia de la lengua dominante a la lengua de herencia en cuanto al uso normativo y la interpretación del artículo definido en plurales definidos y plurales escuetos ${ }^{1}$ en estudiantes universitarios que estaban por ingresar al programa de español como lengua de herencia, específicamente en plurales contables (vasos, lápices, caracoles) y singulares no contables (vino, pan, azúcar).

La transferencia ha sido definida como la incorporación de características de una lengua en otra lengua (Silva Corvalán, 1994: 4). Algunos factores que influyen en la dirección de la transferencia son la difusión estructural (Winford, 2003), el grado de bilingüismo y el contexto social (Weinreich, 1966; Thomason \& Kaufman 1988; Silva Corvalán, 2001). En este caso, siendo el inglés la lengua hegemónica en los Estados Unidos, la transferencia suele ocurrir desde el

1 Un nombre escueto es aquel nombre común que no está constituido por un artículo ni por ningún otro determinante. 
sistema lingüístico del inglés al sistema lingüístico del español —aunque también puede ocurrir en la dirección contraria-, debido a las presiones de la lengua dominante sobre la minoritaria.

Silva Corvalán (1994) considera cuatro fenómenos en los que la transferencia de una lengua a otra puede ocurrir: a) préstamos, b) extensión semántica o reducción de función, c) mayor uso de una forma a expensas de otra y d) eliminación de categorías obligatorias. El tipo de transferencia que se puede encontrar en la interpretación de nombres singulares no contables y plurales contables, y el que atañe a este estudio, es el que corresponde a la extensión semántica o reducción de función, la cual define como la incorporación del significado de una forma $\mathrm{X}$, perteneciente a una lengua $\mathrm{A}$, en una forma $\mathrm{Y}$, perteneciente a una lengua B. La Figura 1 ilustra este mecanismo.
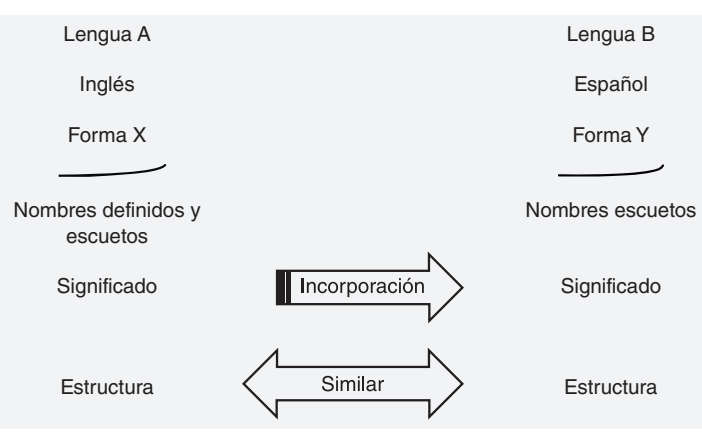

FiguRa 1. Descripción gráfica de la transferencia denominada extensión semántica o reducción de función (basado en Silva Corvalán, 1994)

En este caso, A representa el inglés, B el español, X los nombres definidos y escuetos del inglés e Y los nombres definidos en español.

La transferencia puede ser negativa o positiva (Bussmann, Trauth \& Kazzazi, 1996: 1212-1213). La transferencia positiva ocurre cuando las dos lenguas comparten elementos cuyo significado y/o posición estructural son cercanos, por lo que se transfiere positivamente tal similitud entre las dos lenguas, como ocurre en las oraciones de $(1 \mathrm{a}-\mathrm{b}){ }^{2}$

2 Véase Anexo B para las abreviaturas de las glosas. 


$\begin{array}{lllll}\text { (1) a. } & \text { La familia es } & \text { feliz. } \\ \text { b. } & \text { The family } & \text { is } & \text { happy. } \\ & \text { la-DET familia-N.s } & \text { es-VC } & \text { feliz-ATR } \\ & \text { 'La familia es feliz.' } & & \end{array}$

La transferencia negativa, también conocida como interferencia, ocurre cuando se adoptan elementos de una lengua en otra lengua, pero dichos elementos no están presentes en esta última, es decir, no hay similitudes entre las dos lenguas. Este tipo de transferencia tiene como resultado la producción de usos no esperados. En (2), la oración en español sigue la estructura del inglés al colocar el verbo al final de la oración:

(2) ?El sábado mis amigos vinieron.

En (2) se presenta una oración que no es del todo agramatical en español, pero sí inusual, ya que en el español normativo el verbo suele posicionarse antes del sujeto. La transferencia negativa puede generar usos no-normativos, algunas veces sistemáticos, y dificulta el desarrollo de un dominio de la lengua similar al de los hablantes nativos (Chrabaszcz \& Jiang, 2014: 352).

En $\S 2$ se describe la variable a estudiar, luego, en $\S 3$ se presenta un recuento de la literatura previa del uso y omisión del artículo definido en sintagmas nominales, posteriormente, en $\S 4$ se presenta la metodología, en $§ 5$, los resultados y finalmente, en $\S 6$, las conclusiones y la discusión.

\section{Los nombres definidos y escuetos: diferencias entre las lenguas}

La variable que se analiza en este estudio son los nombres con función argumental en posición preverbal y sus diferentes interpretaciones en inglés y en español. Específicamente, se verá el caso de los nombres plurales contables y singulares no contables escuetos en inglés y definidos en español.

Chierchia (1998) explicita las siguientes diferencias entre las lenguas con respecto a los nombres argumentales:

a) En lenguas germánicas (inglés (3a)) y romances (español (3b)) no puede haber argumentos singulares sin determinante con sustantivos contables: 
(3) a. *Mockingbird sings the whole day.

ruiseñor-N.S canta-V el entero día-CC

'El ruiseñor canta todo el día.'

b. *Perro duerme todo el día.

dpg-N.s sleeps-V whole the day-CC

'The dog sleeps the whole day.'

b) Los argumentos con plurales contables y singulares no contables escuetos son gramaticales en las lenguas germánicas (inglés), pero pueden ser agramaticales (francés) o pueden tener una distribución muy limitada, además de tener una interpretación distinta (español, italiano) en las lenguas romances, ${ }^{3}$ como se ejemplifica en (4-7):

(4) Inglés
a. Cats
sleep
a lot.
gatos-N.pl duermen-V mucho-CC
'Los gatos duermen mucho.'
b. Love is essential.
Amor-N.nc es-VC esencial-ATR
'El amor es esencial.'

(5) Francés
a. *Chats dorment beaucoup. gatos-N.pl duermen-V mucho-CC
'Los gatos duermen mucho.'
b. *Amour est essentiel. amor-N.nc es-VC esencial-ATR
'El amor es esencial.'

3 En español, los sujetos compuestos o en posición posverbal no resultan en usos no-normativos, es el caso de los que están acompañados por algún modificador y los que aparecen en títulos y refranes, como en Carros y motocicletas circulan por la vía pública; Gatos adorables en adopción; Camarón que se duerme, se lo lleva la corriente. 
(6) Italiano

a. *Gatti dormono molto.

gatos-N.pl duermen-V mucho-CC

'Los gatos duermen mucho.'

b. *Amore è essenziale.

amor-N.nc es-VC esencial-ATR

'El amor es esencial.'

(7) Español

a. *Gatos duermen mucho.

b. *Amor es esencial.

c) Para la lectura genérica, las lenguas romances utilizan el artículo definido, pero esto no ocurre en inglés, como se observa en (8).

(8) a. Las tortugas comen algas. (lectura genérica)

The-DET turtles-N.pl eat-v seaweed-OD

'Turtless eat seaweed.'

b. ?The turtles eat seaweed. (lectura genérica)

las-DET tortugas-N.pl comen-V algas-OD

'Las tortugas comen algas.'

d) Tanto las lenguas romances como las germánicas admiten lecturas genéricas o de clase (kind) con el artículo definido singular, como en (9).

(9) a. El perro es el mejor amigo del hombre. (lectura genérica)

The-DET dog-N.S is-VC man's best friend-PRED

The dog is man's best friend.'

b. The $\operatorname{dog}$ is man's best friend.

el-DET perro-N.s es-VC del hombre mejor amigo-PRED

'El perro es el mejor amigo del hombre.'

La oración en (8a) es semánticamente ambigua, pues, además de la lectura genérica, también pude tener una lectura específica, es decir, puede hacer referencia 
a la clase de las tortugas (genérico) o solamente a algunas tortugas (específico). Este uso del artículo definido en (8a) es lo que Vergnaud y Zubizarreta (1992) denominan uso expletivo (expletive) del artículo definido en francés - cuya función es la misma en español: sin contenido denotativo. La diferencia entre el inglés y el español, en este caso, es el estatus del artículo definido: en español puede funcionar como una forma expletiva en donde no otorga referencialidad, carece de contenido denotativo, pero sí tiene una función sintáctica que permite la gramaticalidad de la oración. En inglés el artículo definido no tiene un uso expletivo, por lo que debe estar ausente cuando se expresa un significado genérico o de clase (Vergnaud \& Zubizarreta, 1992: 635), pues, en dicha lengua siempre lleva un contenido denotativo y siempre otorga referencialidad. Por consiguiente, la ausencia de determinante genera una lectura de clase con interpretación genérica (Dayal, 2004), como en (10).

(10) Painters are working (lectura genérica).

pintores-N.pl están trabajando-GV

'Hay pintores trabajando'. (Lit. 'Los pintores están trabajando.')

Cabe mencionar que estas construcciones no siempre tienen una interpretación genérica, sino que, en algunas ocasiones, se les puede dar una interpretación existencial, como la que aparece en (11a) (Carlson, 1977). Lo mismo se puede decir de los singulares no contables, como se observa en (11b).

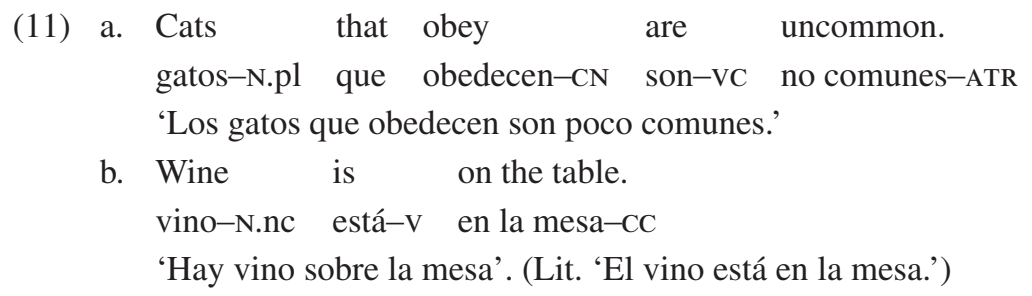

En lo que respecta a los nombres no contables, tanto en inglés como en español, estos tienen una interpretación genérica. Al igual que los nombres plurales contables, los singulares no contables en inglés pueden aparecer sin determinante en posición de sujeto, mientras que en español resultan agramaticales en 
la misma posición (Snape, García Mayo \& Gürel, 2013: 5-6), como se muestra en (12a-b).
a. Wine is good. (lectura genérica)
vino-N.nc es-VC bueno-ATR
'El vino es bueno.'
b. *Vino es bueno. (lectura genérica o específica)

En (12a), el sustantivo wine tiene una interpretación genérica, hace referencia a todos los referentes que pueda tener el vino. En español, oraciones como (13a-b) pueden tener una interpretación genérica o una específica (Snape et al., 2013), El vino en (13a) puede referirse a todos los vinos en general o a un vino en particular, igualmente, en (13b) La información puede referirse a la información en general o a una información específica.
a. El
vino es bueno.
the-DET wine-N.nc is-VC good-ATR
'The wine is good.'
b. La información tiene un precio. the-DET information-N.nc has- $\mathrm{V}$ a price-OD 'The information has a price.'

En resumen, los nombres escuetos en inglés proporcionan una lectura genérica, mientras que los nombres determinados preferencialmente otorgan una lectura específica. En español, los nombres definidos pueden proporcionar tanto una lectura genérica como una específica, mientras que los nombres escuetos tienen una distribución muy limitada. Debido a estas diferencias entre las lenguas, es probable que el sistema gramatical de los hablantes de español como lengua de herencia esté modificado y se encuentre variación del uso normativo del artículo definido en sujetos preverbales. Con la finalidad de reducir la interferencia del inglés al español y de suscitar un mayor número de usos normativos, los hablantes de español como lengua de herencia deben comprender tales diferencias y aprender que los sujetos preverbales con determinante en español pueden interpretarse como genéricos o específicos y no solo como específicos, como sucede 
con el inglés. En otras palabras, deben adquirir una nueva interpretación. Asimismo, deben estar conscientes de que en español los nombres escuetos tienen una distribución más restringida que en inglés y que una de estas restricciones es la ocurrencia de sujetos preverbales escuetos.

A continuación se resume en el Cuadro 1 los usos normativos y no-normativos, así como las posibles interpretaciones de los nombres preverbales escuetos y definidos en español e inglés.

Cuadro 1. Usos normativos y no-normativos de los sujetos preverbales escuetos y definidos con su interpretación en español e inglés

\begin{tabular}{|c|c|c|}
\hline & Escueto & DeFINIDO \\
\hline Inglés & $\begin{array}{l}\text { Uso no-normativo } \\
\text { Sg: *Cat sleeps the whole day (N/A). } \\
\text { Uso normativo } \\
\mathrm{Pl} \text { : Cats sleep the whole day (Gen.). } \\
\text { NC: Wine is good (Gen.). }\end{array}$ & $\begin{array}{l}\text { Uso normativo } \\
\text { Sg: The cat sleeps the whole day (Esp.). } \\
\text { Pl: The cats sleep the whole day (Esp.). } \\
\text { Nc: The wine is good (Esp.). }\end{array}$ \\
\hline Español & $\begin{array}{l}\text { Uso no-normativo } \\
\text { Sg: *Gato duerme todo el día (N/A). } \\
\text { Pl: *Gatos duermen todo el día (N/A). } \\
\text { NC: *Vino es bueno (N/A). }\end{array}$ & $\begin{array}{l}\text { Uso normativo } \\
\text { Sg: El gato duerme todo el día (Esp.). } \\
\text { Pl: Los gatos duermen todo el día (Esp./Gen.). } \\
\text { Nc: El vino es bueno (Esp./Gen.). }\end{array}$ \\
\hline
\end{tabular}

Nota: No aplica (N/A), singular (Sg), plural (PI), no contable (NC), genérica (Gen) y específica (Esp).

\section{Estudios previos}

El uso del artículo definido en sintagmas nominales en inglés y en español como L1 y L2 se ha estudiado en la producción de niños y adultos, y, recientemente, en las lenguas de herencia. Un estudio que analiza las diferencias entre las lenguas en torno a la interpretación del artículo definido y de plurales escuetos en hablantes nativos de inglés y español es el de Pérez Leroux, Munn, Schmitt y DeIrish (2004). Estos investigadores estudiaron la adquisición de plurales definidos en contextos genéricos en niños de edad preescolar monolingües en inglés y en español, así como en un grupo de adultos monolingües en inglés. Encontraron que los niños hablantes nativos de inglés interpretaron los plurales definidos como genéricos hasta un $70 \%$ de las veces. Esto no ocurrió con el grupo de adultos, dado que esta interpretación del artículo definido no está disponible en 
inglés. El grupo de niños hablantes nativos de español prefirió la interpretación genérica de los plurales definidos (80-95\%) a pesar de que la interpretación específica también es una opción gramatical viable en español. Esta investigación muestra que a temprana edad tanto la interpretación genérica como la específica de los plurales definidos están presentes en ambas lenguas.

Otro estudio que también incluye a hablantes de inglés como primera lengua es el de Gelman y Raman (2003). Estas investigadoras examinaron la distinción genérico/específico por medio del artículo definido y de los plurales escuetos en niños de 4 años de edad y adultos. Ambos grupos de participantes mostraron una actuación esperada: una interpretación genérica para los nombres escuetos y una específica para aquellos que tenían el determinante definido. Sin embargo, la presencia o ausencia del determinante tuvo mayor efecto entre los adultos, pues este grupo seleccionó la interpretación esperada con mayor frecuencia. Los resultados evidencian que tanto los niños como los adultos tienen una interpretación similar de los sintagmas nominales genéricos y específicos en inglés.

Pasando a los estudios que incluyen participantes de segunda lengua, Cuza, Guijarro Fuentes, Pires y Rothman (2012) investigaron hasta qué punto hablantes nativos de inglés adquieren la restricción de los plurales escuetos en español (gatos vs los gatos) en aprendices universitarios de español avanzado como segunda lengua y hablantes nativos de español. Los resultados revelaron que los hablantes nativos aceptaron la lectura específica y genérica en nombres determinados por el artículo definido y reconocieron la agramaticalidad de las frases escuetas. El grupo avanzado también aceptó las dos lecturas del artículo definido, sin embargo, aceptaron como gramaticales un cuarto de las frases escuetas en contextos genéricos.

Ionin, Montrul y Crivos (2013) investigaron la transferencia de la lengua materna en la gramaticalidad e interpretación de plurales en español e inglés. Para el estudio del inglés, participaron aprendices de inglés cuya lengua materna es el español, así como un grupo de hablantes nativos. Igualmente, en el caso del estudio del español, participaron aprendices de español cuya lengua materna es el inglés, así como hablantes nativos. Todos los participantes eran adultos. Los aprendices de segunda lengua fueron divididos en tres niveles: principiante, intermedio y avanzado. Los resultados de la prueba de interpreta- 
ción, en lo referente al uso de los plurales, mostraron transferencia de la primera lengua en ambas direcciones, por lo menos en los niveles principiantes, dado que los niveles avanzados se comportaron como los hablantes nativos. Los resultados de la prueba de aceptabilidad en inglés — la cual medía la gramaticalidad - mostraron que todos los grupos otorgaron una mayor aceptación a los plurales definidos (The tables are round) en comparación con los plurales escuetos (Tables are round) en la categoría de plurales específicos. Por el contrario, todos los grupos prefirieron la aceptación de los plurales escuetos (Tables have four legs) que la de los plurales definidos (The tables have four legs) en la categoría de plurales genéricos.

Las autoras encontraron que mientras la competencia lingüística aumenta, la aceptabilidad de los plurales definidos se incrementa, mientras que la de los plurales escuetos disminuye. En lo referente a la prueba en español, los resultados mostraron que los tres grupos otorgaron un mayor grado de aceptabilidad a los plurales definidos en ambas categorías: específica y genérica. También observaron que según aumentaba la competencia en la lengua, el grado de aceptabilidad de los plurales definidos era mayor, mientras que el de los plurales escuetos era menor.

En lo referente a estudios con hablantes de español como lengua de herencia, recientemente dos estudios analizaron la interpretación del artículo definido en esta población. Montrul e Ionin (2010) estudiaron la gramaticalidad, interpretación y posición in/alienable ${ }^{4}$ del artículo definido en hablantes de español como lengua de herencia. Los resultados del experimento mostraron un índice alto $(86 \%)$ de respuestas esperadas en cuanto a la gramaticalidad de los sintagmas nominales. Asimismo, encontraron transferencia en la interpretación genérica del artículo definido, pero no en los contextos de posesión inalienable. Las investigadoras creen que este fenómeno se debe no solamente al input reducido y a la falta de uso sino también a la transferencia morfosintáctica y semántica del inglés (Montrul \& Ionin, 2010: 450). Concluyen que en este tipo de transferencia tiene más relevancia la lengua dominante que la edad de adquisición.

4 Por ejemplo, en la oración Marcos levanta la mano, puede referirse a su mano (inalienable) o la mano de alguien más (alienable). 
Dos años más tarde, Montrul e Ionin (2012) replicaron su estudio de 2010, pero esta vez incluyeron un grupo de angloparlantes. Ambos grupos mostraron transferencia en la interpretación genérica del artículo en español, mientras que solamente el grupo de aprendices de español como segunda lengua mostraron signos de transferencia en la posesión inalienable. Las autoras concluyeron que la transferencia de la lengua dominante sobre la minoritaria, además de las condiciones de input reducido, influye en la formación de las gramáticas de los estudiantes adultos — sean de segunda lengua o de lengua de herencia — y no así, la edad de adquisición de la lengua.

En conclusión, la literatura existente sobre el dominio del artículo definido y su interpretación en sintagmas nominales ha arrojado resultados similares en diversas lenguas y en diversos grupos de participantes (primeras y segundas lenguas, lengua de herencia). En general, los hablantes nativos prefieren la interpretación genérica del artículo definido sobre la específica. Los estudiantes de español como segunda lengua y los hablantes de español como lengua de herencia han mostrado que son capaces de adquirir las restricciones de uso de los nombres escuetos y de identificar la interpretación genérica y específica, ambas disponibles en español. No obstante, ninguno de los estudios previamente mencionados investiga el uso de nombres en cuanto a su clasificación como contables y no contables. Esta investigación pretende contribuir al estudio de los sintagmas nominales en posición de sujeto al indagar en el uso sintáctico y la interpretación semántica de nombres tanto contables (plurales) como no contables (singulares).

Las preguntas de investigación a las que se busca dar respuesta son las siguientes: 1) ¿Hay transferencia del inglés al español en hablantes de herencia en cuanto al uso del artículo definido en la producción escrita de nombres plurales contables y singulares no contables?; 2) ¿hay transferencia del inglés al español tanto en contextos genéricos como en contextos específicos?; 3) ¿qué tipo de nombres - plurales contables o singulares no contables - representan un mayor desafío para los hablantes de español como lengua de herencia en cuanto al uso esperado del artículo definido?; 4) ¿hay una correlación entre el dominio del uso normativo de la variable y la competencia linguiística de los participantes según su ubicación en los diversos niveles de escolaridad?

Se espera que los participantes presenten variación en el uso del artículo definido y transfieran, en algunos casos, la estructura y el significado que se da a 
los sujetos preverbales en inglés, debido a la influencia de la lengua dominante sobre la lengua de herencia, como se ha mostrado en estudios anteriores (Montrul \& Ionin, 2010, 2012). Aunque también se prevé que el uso y la exposición a la lengua de herencia contribuyan a reducir dicha transferencia, especialmente en cuanto al uso normativo del artículo definido. Asimismo, se presume que la transferencia es influida tanto por la semántica como por la sintaxis de la lengua dominante. Pese a todo lo anterior, también puede suponerse que el hecho de que exista un número limitado de restricciones para los nombres escuetos en posición preverbal en español (para contextos permitidos véase la nota 3) puede hacer que la adquisición de los usos sintácticos sea más fácil para los hablantes de español como lengua de herencia en comparación con la adquisición de las diferentes lecturas de los nombres plurales.

\section{Metodología}

\subsection{Participantes}

Los participantes de este estudio eran estudiantes de la Universidad de Houston matriculados en distinto año de escolaridad. Al momento de realizar la composición, aspiraban a tomar una clase de español en el programa de español como lengua de herencia de la misma universidad. El nivel de conocimiento en español se determinó mediante los resultados del examen de ingreso a dicho programa. Los participantes se dividieron en cuatro grupos según la evaluación de su competencia lingüística: principiante, intermedio-bajo, intermedio-alto y avanzado. Se seleccionó aleatoriamente un total de 200 composiciones: 50 composiciones por cada grupo.

\subsection{Instrumento}

Las composiciones seleccionadas constituían una sección del examen de ubicación del programa de lengua de herencia. Antes de realizar la composición, los participantes debían leer un artículo argumentativo sobre la privacidad en las redes sociales y en la Web 2.0; la extensión de esta lectura era de una página. Posteriormente, se les daba 30 minutos para escribir un ensayo sobre el tema pro- 
puesto, en el que debían opinar a favor o en contra de los argumentos expuestos por el autor del ensayo que leyeron.

\subsection{Análisis de los datos}

De las 200 composiciones — clasificadas en 4 grupos de 50 ensayos cada uno-, no todas presentaron nombres preverbales en posición de sujeto determinados por el artículo definido o nombres escuetos sin ningún otro modificador, por lo que se analizaron solamente las composiciones que sí contaban con nombres que cumplían dicha descripción: 164 en total. El Cuadro 2 muestra el número de composiciones que presentaron la variable, desglosando el mínimo, el máximo, el promedio y el total de sintagmas nominales por grupo.

Cuadro 2. Producción por grupos de composiciones. Desglose de usos mínimos, máximos y promedio por composición

\begin{tabular}{lccccc}
\hline \multirow{2}{*}{ Grupo } & Comps. con SSNN & \multicolumn{3}{c}{ Uso POR Composición } & \multirow{2}{*}{ Total de SSNN } \\
\cline { 3 - 5 } & & Mín. & Máx. & Promedio & 203 \\
\hline Principiantes & 41 & 1 & 10 & 4.95 & 288 \\
Intermedio bajo & 47 & 1 & 11 & 6.13 & 171 \\
Intermedio alto & 34 & 1 & 11 & 5.03 & 233 \\
Avanzado & 42 & 1 & 13 & 5.55 & 895 \\
Total & 164 & & & & \\
\hline
\end{tabular}

Cabe señalar que solamente se analizaron nombres en posición de sujeto preverbal y se excluyeron los nombres propios y los pronombres. Tampoco se contabilizaron nombres precedidos por demostrativos, posesivos o cuantificadores, ya que, si bien ocupan la misma posición que el artículo definido dentro del sintagma nominal, están fuera del alcance de este estudio. También se excluyeron los préstamos del inglés al español y palabras como Facebook, Twitter, web.

Los nombres se clasificaron de la siguiente manera: a) según el uso u omisión del artículo definido, b) según los contextos en los que aparecían — genérico o específico- - y c) según el tipo de nombre — plurales contables o singulares no contables. Luego se realizó un análisis estadístico de los datos usando el 
programa Statistical Package for the Social Sciences (SPSS) con el fin de encontrar las diferencias entre las variantes y los grupos.

\section{Resultados}

Esta sección presenta los resultados del estudio conforme a las preguntas de investigación, así como algunos ejemplos de la producción de los participantes (en el Anexo A se muestra una composición completa de un participante de cada nivel). ${ }^{5}$

La primera pregunta de investigación cuestionaba si había transferencia del inglés al español en cuanto al uso del artículo definido en la producción escrita de los nombres plurales contables y singulares no contables; la respuesta es afirmativa. En el Cuadro 3 se muestra el total de sintagmas nominales escuetos y definidos por grupo. Como se puede observar, la omisión del artículo definido va disminuyendo según avanza la competencia lingüística de los participantes en la lengua de herencia.

CuAdRo 3. Producción de nombres escuetos (NNEE), nombres definidos (NNDD) y total de sintagmas nominales (SSNN) por grupo

\begin{tabular}{|c|c|c|c|c|c|c|}
\hline \multirow{2}{*}{ Grupo } & \multicolumn{2}{|c|}{ NNEE } & \multicolumn{2}{|c|}{ NNDD } & \multicolumn{2}{|c|}{ TOTAL SSNN } \\
\hline & $n$ & $\%$ & $n$ & $\%$ & $n$ & $\%$ \\
\hline Principiantes & 151 & 74 & 52 & 26 & 203 & 100 \\
\hline Intermedio bajo & 207 & 72 & 81 & 28 & 288 & 100 \\
\hline Intermedio alto & 152 & 89 & 19 & 11 & 171 & 100 \\
\hline Avanzado & 206 & 88 & 27 & 12 & 233 & 100 \\
\hline Total & 716 & & 179 & & 895 & \\
\hline
\end{tabular}

La segunda pregunta buscaba responder en qué tipo de interpretación habría mayor transferencia. Se encontró transferencia en todos los niveles en ambos tipos de nombres en la interpretación genérica del artículo definido, mientras que en la específica se encontró transferencia solamente en el grupo de principiantes. A continuación se presentan ejemplos de la producción de los participantes. En (14a-b), se han resaltado en itálicas los nombres singulares no contables escue-

5 Las composiciones se han transcrito tal como fueron redactadas por los participantes. 
tos en contextos genéricos. En (14c), se presenta el mismo tipo de nombre, pero en un contexto específico. Los nombres en itálicas en (15a-c) son plurales contables escuetos en contextos genéricos. ${ }^{6}$

(14) a. Como comentaron en el artículo anterior, *información personal no tiene que ser percibido como una "mina de oro" sino como un puente hacia otras personas. [avanzado, 418, M]

b. Para concluir, aunque *privacidad parezca muy importante a la gente, no es la verdad en el internet. [intermedio-alto, 302, H]

c. Esa es el unico manera de estar seguro que *informacion que no quieres que sea publico, se queda asi. [principiante, $117, \mathrm{H}$ ]

(15) a. Por ese razón *niños neccesitan algien como su madre y padre para asistir o, en tiempo, quitar su informaccion personal del internet. [intermedio-alto, 127, M]

b. Sabiendo los riesgos y que hacer hayuda que *usuarios tengan precaucion antes de usar la red. [principiante, 202, M]

c. Es otra manera que *agencias buscan a sus clientes prometiendo guardar este tipo de información. [avanzado, 403, M]

El hecho de que la omisión del artículo definido haya sido escasa en los contextos específicos está relacionado con que, en ambas lenguas, la interpretación específica se encuentra presente en la referencialidad que otorga el artículo definido, lo cual posibilita una transferencia positiva debido a la convergencia entre los dos sistemas lingüísticos. Que el grupo de principiantes se haya desviado de la norma en los contextos específicos probablemente se deba a una inseguridad lingüística: la composición de un ensayo en español es una tarea que, posiblemente, los participantes nunca o muy pocas veces habían realizado.

En cambio, en la interpretación genérica, en donde la forma y el significado no son equivalentes en las dos lenguas, hay mayor oportunidad para que la

6 Los ejemplos se han transcrito tal como se encuentran en las composiciones. En las etiquetas de cada ejemplo se presenta primeramente el nivel de dominio del participante $(1=$ principiante, 2 = intermedio-bajo, 3 = intermedio-alto y 4 = avanzado), las dos cifras siguientes corresponden al número que le corresponde y la inicial (M o F) a su género. Así, por ejemplo, en (14), el número 418 se refiere a la participante mujer número 18 del grupo avanzado. 
transferencia ocurra. Por ello los hablantes de español como lengua de herencia ocasionalmente omiten el artículo definido en este tipo de construcciones, como se ejemplifica en (16a-b) con fotografías y mujeres; pues en inglés los plurales escuetos tienen una interpretación genérica. Obsérvese que este tipo de interferencia es una variación de la norma, pues en (16a) mundo aparece determinado, lo mismo ocurre con hombres en (16b).

(16) a. Cuando termine abriendo una cuenta el mundo sabe su nombre, su edad, ciudad de nacimiento, y mucho mas. Fotographias se pierden en la red de los medios sociales. [intermedio-bajo, 212, H]

b. Mucha gente se preocupa por su privacidad. Se pasan su vida tratando de mantener su vida privada. Mujeres se preocupan por perder su bolsa y los hombres se preocupan por perder su cartera. [avanzado, 404, M]

En la Figura 2 se observan los resultados de las interpretaciones genérica y específica. En lo que respecta a los nombres con lectura genérica, el grupo de principiantes mostró resultados esperados en un 66\% (92/139), el grupo intermedio-bajo en un $61 \%$ (120/196), el grupo intermedio-alto en un 84\% (98/116) y el grupo avanzado en un $81 \%$ (111/137). Para la lectura específica, el grupo de principiantes manifestó resultados esperados en un $79 \%$ (15/19), y los tres grupos restantes mostraron un

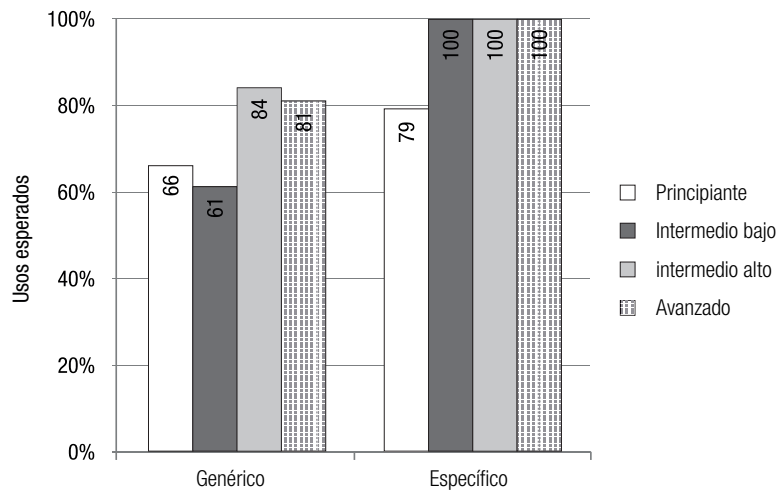

Figura 2. Porcentaje de usos esperados de los nombres en contextos genéricos y específicos por grupo 
$100 \%$ (11/11 para el grupo intermedio-bajo, 11/11 para el grupo intermedio-alto y $8 / 8$ para el grupo avanzado) de respuestas esperadas.

La tercera interrogante indagaba sobre la susceptibilidad de la transferencia según el tipo de nombres (plurales contables/singulares no contables). Los resultados revelan una mayor transferencia en los plurales contables en los cuatro grupos, como se observa en el Cuadro 4, a pesar de que el número de este tipo de nombres encontrados en las composiciones fue menor en todos los grupos en comparación con el número de nombres singulares no contables.

CuADRo 4. Número de casos esperados (n), total de ocurrencias (t) y porcentaje de plurales contables y singulares por grupo

\begin{tabular}{lccccc}
\hline \multirow{2}{*}{ Grupo } & \multicolumn{2}{c}{ PLURALES CONTABLES } & & \multicolumn{2}{c}{ SinGulARES NO CONTABLES } \\
\cline { 2 - 3 } \cline { 5 - 6 } & $\mathrm{n}(\mathrm{t})$ & $\%$ & & $\mathrm{n}(\mathrm{t})$ & $\%$ \\
\hline Principiantes & $31(50)$ & 62 & & $76(108)$ & 70 \\
Intermedio bajo & $37(66)$ & 56 & & $74(141)$ & 67 \\
Intermedio alto & $35(46)$ & 76 & & $78(91)$ & 91 \\
Avanzado & $41(54)$ & 76 & & $781)$ & 86 \\
\hline
\end{tabular}

La Figura 3 permite visualizar las diferencias porcentuales entre los plurales contables y los singulares no contables según su lectura genérica o específica. Solamente el grupo de principiantes tuvo dificultades con la interpretación específica. De nuevo, los resultados muestran un menor dominio de los usos esperados en los nombres en contextos genéricos que en contextos específicos, lo cual corrobora que hay mayor transferencia en estos contextos debido a la falta de correspondencia sintáctico-semántica entre el inglés y el español.

La respuesta a la cuarta pregunta, sobre la influencia del uso y exposición de la lengua de herencia en el uso normativo de la variable, es que, aunque parece haber un mayor dominio de las variables según aumenta el nivel de manejo del español de los participantes, no se encontraron diferencias significativas entre los grupos en ninguna de las variables según el resultado de la chi-cuadrada y los análisis de varianza. Se realizaron pruebas de asociación chi-cuadrada entre las dos interpretaciones y los dos tipos de palabras — plurales contables y singulares no contables, excepto entre plurales contables y la interpretación específica debido a que las frecuencias eran menores a cinco. En los demás casos todas las frecuen- 


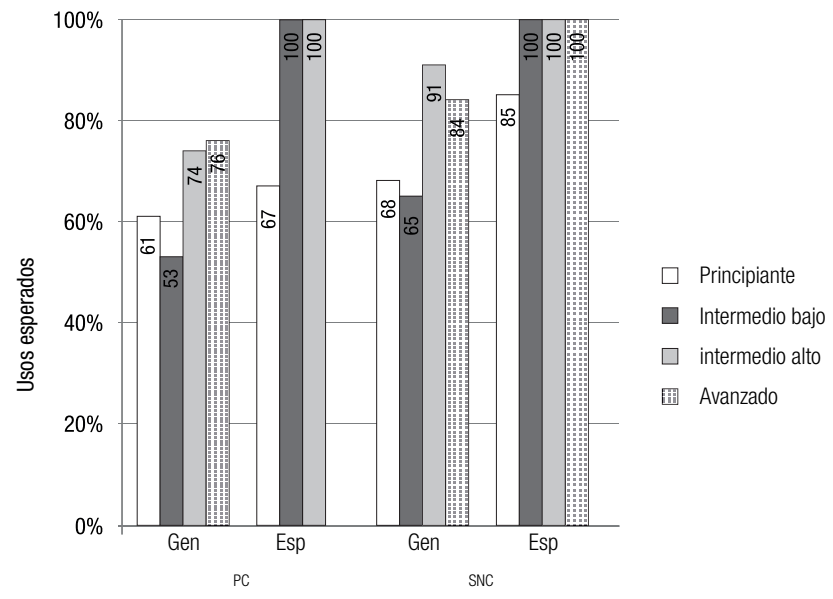

Figura 3. Porcentaje de usos esperados en plurales contables (PC) y singulares no contables (SNC) en contextos genéricos (Gen) y específicos (Esp) por grupo

cias esperadas excedían este número. Los resultados de la chi-cuadrada entre la interpretación genérica y los plurales contables son $\chi^{2}(3)=3.320$, $p=.344$; los de la relación entre la interpretación genérica y los singulares no contables son $\chi^{2}(3)=5.847, p=.119$ y los de la relación entre la interpretación específica y los singulares no contables son $\chi^{2}(3)=0.206, p=.997$. Asimismo, las diferencias entre los grupos y entre los tipos de nombres y su interpretación no fueron estadísticamente significativos, tanto en los resultados del análisis simple de varianza (one-way ANOVA) como en las pruebas de comparación posteriores (post hoc) de Bonferroni. Es posible que el tipo de actividad abierta haya repercutido en los resultados. A excepción de la lectura específica, no hay un dominio total de uso del artículo definido en ningún nivel.

Esto significa que los hablantes de español como lengua de herencia, por ser el inglés su lengua dominante, poseen dificultades con la producción del artículo definido sin importar que tengan diferente competencia lingüística de la lengua de herencia, excepto con nombres que aparecen en contextos específicos, en los cuales solamente el grupo principiante reportó casos no esperados. 


\section{Discusión y conclusiones}

Esta investigación se planteó como objetivo averiguar si en el español de hablantes de lengua de herencia había transferencia —en la dirección del inglés al español- de los usos normativos y la interpretación de los sintagmas nominales determinados y escuetos. Según los resultados anteriores, se observó mayor transferencia en la lectura genérica que en la específica debido a las diferencias entre las lenguas. Se cree que esto ocurre porque la interpretación genérica en inglés está presente en los nombres escuetos, mientras que, en español, a los nombres definidos se les otorga una lectura genérica preferencial sobre la lectura específica. Por otro lado, el artículo definido, en inglés y en español, otorga una lectura específica por medio de su capacidad de referencia, por lo cual hay una transferencia positiva en los nombres definidos específicos. Los resultados revelan que los hablantes de español como lengua de herencia de esta muestra omiten el artículo definido en sintagmas nominales preverbales en todos los niveles de competencia en la lengua de herencia, excepto en los nombres con interpretación específica. Finalmente, no se encontraron diferencias significativas entre las variables ni entre los grupos, lo cual sugiere que la transferencia se debe a la influencia de la lengua dominante (Montrul \& Ionin, 2010, 2012) y que dicha influencia, si bien disminuye conforme avanza la competencia en la lengua de herencia, está presente en todos los participantes sin importar el nivel de competencia que posean.

En lo referente a la distinción plurales contables y singulares no contables, a pesar de que se encontró que los participantes produjeron aproximadamente el doble de nombres singulares no contables en comparación con los plurales contables, hubo una mayor omisión del artículo definido en los plurales contables en todos los niveles. Se encontró interferencia del inglés al español tanto en los plurales contables como en los singulares no contables en la lectura genérica del artículo definido, principalmente. Los contextos genéricos resultaron problemáticos para todos los grupos, en cambio, en el contexto específico hubo muy poca interferencia del inglés: solo el grupo principiante mostró algunas omisiones del artículo definido en este contexto.

Se espera que con una mayor exposición (recepción) y uso de la lengua (producción), en conjunto con la enseñanza explícita de las variables en los 
contextos analizados, los casos no esperados se reduzcan hasta desaparecer y que los hablantes de español como lengua de herencia adquieran la interpretación genérica en los nombres definidos. En este caso, la dificultad del manejo de este tipo de nombres puede deberse a lo que White (2003) denomina pobreza de estímulo, es decir, a una disparidad entre la exposición (input) y la producción (output) de la variable, donde la producción puede ser la no esperada debido a la falta de exposición.

Entre las limitaciones de este estudio está la falta de uso de otros instrumentos con los cuales se puedan contrastar los resultados obtenidos, por lo que se recomienda que en investigaciones futuras se implementen instrumentos adicionales para medir las variables lingüísticas desde otros enfoques, así como para medir la interpretación de los sintagmas nominales (pruebas de veracidad, pruebas de gramaticalidad, traducciones). También convendría integrar instrumentos para medir el efecto de la enseñanza en estas variables con pruebas de tratamiento previo (pre-test) y posterior (pos-test), así como la realización de estudios longitudinales (tiempo aparente) en los que se comparen varios niveles de escolaridad.

Estudios anteriores (Ionin et al., 2013) sugieren que el uso sintáctico y la interpretación del artículo definido requieren de esfuerzos cognitivos diferentes, debido a que las restricciones sintácticas de gramaticalidad son más fácilmente superadas que las diferencias semánticas (interpretación). En el ámbito pedagógico, esto significa que los hablantes de español como lengua de herencia deben aprender la restricción de que un sujeto preverbal no puede aparecer sin determinante en español, lo cual es cognitivamente más sencillo que adquirir una interpretación del artículo definido que no está presente en inglés, es decir, la interpretación genérica en plurales contables y singulares no contables definidos.

No obstante, como se ha observado en otros estudios (Slabakova, 2006; Montrul \& Ionin, 2010, 2012; Ionin et al., 2013;) y como se muestra en los resultados, los usos no esperados que producen los aprendices se pueden superar con una mayor exposición a la lengua, especialmente los usos no-normativos sintácticos, por ser más evidentes. Como señalan Ionin et al. (2013), los aprendices de segunda lengua no permanecen restringidos a la gramática de la lengua materna, sino que superan las diferencias entre las dos lenguas según avanza el nivel de competencia. Lo mismo se puede decir de los hablantes de lengua de herencia: a mayor exposición a la lengua de herencia, menor transferencia de la 
lengua dominante. Para superar las diferencias entre las lenguas de los aspectos sintáctico y semántico del artículo definido en español, se recomienda que en el aula se incluyan actividades que presenten explícitamente las diferencias entre las dos lenguas, es decir, las diferentes interpretaciones de los sintagmas nominales según el tipo de nombres y el número gramatical en ambas lenguas, así como las restricciones sintácticas de los nombres escuetos en español.

\section{Referencias}

Austin, Jennifer; Blume, María, \& Sánchez, Liliana (2015). Bilingualism in the Spanish-speaking world. Linguistic and cognitive perspectives. Cambridge: Cambridge University Press.

Benmamoun, Elabbas; Montrul, Silvina, \& Polinsky, Maria (2013). Defining an "ideal” heritage speaker: Theoretical and methodological challenges reply to peer commentaries. Theoretical Linguistics, 39(3-4), 1-56. Recuperado de https://dash.harvard.edu/bitstream/ handle/1/11856180/benmamoun_definingideal.pdf?sequence=1

Bussmann, Hadumod; Trauth, Gregory, \& Kazzazi, Kerstin (1996). Routledge dictionary of Language and Linguistics. Londres: Routledge.

Carlson, Gregory Norman (1977). Reference to kinds in English (Tesis doctoral). University of Massachusetts, Amherst. Recuperado de https://search.proquest.com/docview/302863258

Chierchia, Gennaro (1998). Reference to kinds across language. Natural Language Semantics, 6(4), 339-405. doi: 10.1023/A:1008324218506

Chrabaszcz, Anna, \& Jiang, Nan (2014). The role of the native language in the use of the English nongeneric definite article by L2 learners: A cross-linguistic comparison. Second Language Research, 30(3), 351-379. doi: 10.1177/0267658313493432

Cuza, Alejandro; Guijarro Fuentes, Pedro; Pires, Acrisio, \& Rothman, Jason (2012). The syntax-semantics of bare and definite plural subjects in the L2 Spanish of English natives. International Journal of Bilingualism, 17(5), 634-652. doi: 1367006911435594

Dayal, Veneeta (2004). Number marking and (in)definiteness in kind terms. Linguistics and Philosophy, 27(4), 393-450. Recuperado de http://www.jstor.org/stable/25001933

Gelman, Susan A., \& Raman, Lakshm (2003). Preschool children use linguistic form class and pragmatic cues to interpret generics. Child Development, 74(1), 308-325. doi: 10.1111/14678624.00537 
Ionin, Tania; Montrul, Silvina, \& Crivos, Mónica (2013). A bidirectional study on the acquisition of plural noun phrase interpretation in English and Spanish. Applied Psycholinguistics, 34(3), 483-518. doi: 10.1017/S0142716411000841

Montrul, Silvina, \& Ionin, Tania (2010). Transfer effects in the interpretation of definite articles by Spanish heritage speakers. Bilingualism: Language and Cognition, 13(4), 449-473. doi: $10.1017 / \mathrm{S} 1366728910000040$

Montrul, Silvina, \& Ionin, Tania (2012). Dominant language transfer in Spanish heritage speakers and second language learners in the interpretation of definite articles. The Modern Language Journal, 96(1), 70-94. doi: 10.1111/j.1540-4781.2012.01278.x

Pérez leroux, Ana Teresa; Munn, Alan; Schmitt, Cristina, \& DeIrish, Michelle (2004). Learning definite determiners: Genericity and definiteness in English and Spanish. En Boston University Conference on Language Development 28 Proceedings Supplement, 1-12. Recuperado de www.bu.edu/bucld/files/2011/05/28-perez-leroux.pdf

Silva Corvalán, Carmen (1994). Language contact and change: Spanish in Los Angeles. Nueva York: Oxford University Press.

Silva Corvalán, Carmen (2001). Sociolingüística y pragmática del español. Washington, D. C.: Georgetown University Press.

Slabakova, Roumyana (2006). Learnability in the second language acquisition of semantics: A bidirectional study of a semantic parameter. Second Language Research, 22(4), 498-523. doi: $10.1191 / 0267658306$ sr277oa

Snape, Neal; García Mayo, María del Pilar, \& Gürel, Ayşe (2013). L1 transfer in article selection for generic reference by Spanish, Turkish and Japanese L2 learners. International Journal of English Studies, 13(1), 1-28. Recuperado de https://eric.ed.gov/?q= EJ1041876\&id=EJ1041876

Thomason, Sarah G., \& Kaufman, Terrence (1988). Language contact, creolization, and genetic Linguistics. Berkeley: University of California Press.

VALDÉs, GuAdALUPE (2000). The teaching of heritage languages: An introduction for Slavic-teaching professionals. En Olga Kagan \& Benjamin Rifkin (Eds.), The learning and teaching of Slavic languages and cultures (pp. 375-404). Bloomington: Slavica.

Vergnaud, Jean-Roger, \& Zubizarreta, Maria Luisa (1992). The definite determiner and the inalienable constructions in French and English. Linguistic Inquiry, 23(4), 595-652. Recuperado de https://www.jstor.org/stable/4178791?seq=1\#page_scan_tab_contents

WeInReich, URIEL (1966). Languages in contact: Findings and problems. La Haya: Mouton. 
White, Lydia (2003). Second language acquisition and universal grammar. Cambridge: Cambridge University Press.

Winford, Donald (2003). An introduction to contact linguistics. Malden: Blackwell.

\section{Anexos}

En este apartado se presenta un ejemplo de la escritura de los participantes por cada nivel. Se han resaltado los sustantivos analizados en cursivas. Los sustantivos escuetos se encuentran precedidos por un asterisco, mientras que los sustantivos definidos se encuentran modificados por el correspondiente artículo.

\section{A. Muestra de composiciones}

Nivel principiante

La privacion en el Internet es algo que no tiene sentido en estos tiempos. Muchos personas hoy dia usan el Internet 24/7, poniendo demasiado tiempo en sitios como Facebook y Twitter. Es en estos sitios que ponen informacion sobre su vida como datos, nombres, y haria de vivir. Estas personas quieren (share) su infomacion personal, y ponerlo en un lugar que cualquier persona, que tiene Internet, puede leer. El Internet es una espacio vitual que todos usan y tienes acceso. Es por esta razon que yo creo que *personas no se deban quejarse de su privacion si usan el Internet. Ellos estan tomando una consciencia accion en poner ese informacion en varias paginas donde no tienen seguridad en control en quien lo pueden leer y acessar. *Personas que se quejan de su prividad faltan que hacer el decision de parar de usar estas sitios. Esa es el unico manera de estar seguro que *informacion que no quieres que sea publico, se queda asi. [principiante, 117, H]

Nivel intermedio-bajo

Estos mismos usuarios despues se molestan por que *compañías coleccion su informacion personal e intentan distibuirla. Hay solo una manera de prevenir que *información personal. La unica manera es evitar registrarse con sitios sociales. Sin que la persona personalmente de su informacion, no hay manera que los sitios la obtengan. En cónclusion, el internet continuara creciendo en los proximos años. Muchos mas sitios sociales seran inventados 
y *companias averiguaran mas maneras para obtener la informacion de usuarios. *Usuarios deben de entender que la mayoria del tiempo. No hay manera de registrarse en sitios sociales, compartir su informacion personal y no pensar que. [intermedio-bajo, 206, H]

Nivel intermedio-alto

* Privacía y el Internet son asuntos de mucha importancia en este mundo interconectado. A mi placer, aunque se necesita herramientas para guardarnos la información personal, es probable que pocos las van a usar. En este día y época, casí todos en el mundo desarollado saben lo que es el internet; muchas celebridades, aún unos políticos, tienen cuentas de Twitter y facebook, con todos los millones de personas compartiendo cualquieras pensamientos que se les parezcan interesante. Al otro mano, esa compartamiento libre de asuntos personales abre la puerta para que *cazadores de inocencia puedan buscar maneras de asustar otros. También es que se pueda afectar más gente con cybercrimen, como todos los millones en facebook. Estoy de acuerdo con el artículo en que cada vez que alguién deja una poste en una red social, se deja más información libre para todos a ver. Creo que, a pesar de que postes de facebook son frivolosos, estas se revelan mucha información. La privacidad es muy importante a casí todos cuando se la están discutiendo, pero, como el artículo dice, las acciones de la gente no se juntan con sus deseos. Aunque hayan herramientas para esconder la información personal de personas desconocidas, la mayoría de la gente no se las usen. Hay varias razones, como «sería más fácil para que mis amigos me alcancen»o «estuve perezosa en no haciéndolo», u otras cosas como el hecho de que facebook y twitter son entretenemientos, como juegos y así no parecen muy importantes. En corto, lo relevante es que para la gente, no les importa mucho la privacia en el internet. Para concluir, aunque *privacidad parezca muy importante a la gente, no es la verdad en el internet. Lo que se necesita hacer es proteger información personal automáticamente, para que la gente no tengan que hacerlo. [intermedio-alto, 302, M]

Nivel avanzado

Mucha gente se preocupa por su privacidad. Se pasan su vida tratando de mantener su vida privada. *Mujeres se preocupan por perder su bolsa y los hombres se preocupan por perder su cartera. La razón que se preocupan es porque en su bolsa ó cartera tienen mucha información personal. Tambien tratan de no incluyír su number telefonico en el catalogo de numeros. El 
problema es que la mayoría de esta gente es parte de programas como Facebook y Twitter. Facebook y twitter son programas que sin querer le ofrece al publico toda la información privada de los miembros. Yo estoy de acuerdo con el al articulo "Privacy and the Internet". $L a$ populación se contradice porque aunque se preocupan por su privacidad a la misma vez se la ofrecen a medio mundo atravez de estos programas. Han habido muchos problemas con esté téma. *Gente a muerto y mucha gente ha sido afectada psycologicamente. Pero los miembros siguen sin entender la importancia de seleccionar la informacion que escriben en estas paginas del internet. El articulo dice muy claramente que los mensajes que se escriben en estas paginas personales muchas veces incluyen direcciones y numeros de teléfono. Ser parte de estas redes no es malo pero se puede convertir en algo peligroso si demasiada informacion es ofrecida sobre mensajes y informacion publicada. Hay un limite que no se puede pasar porque si ese limite es cruzado entonces pueden haber problemas. Yo pienso que *adultos pueden entender este tema mas que *adolecentes y por eso es importante que los padres vigilen las paginas de sus hijos. *Adolecentes quieren ser popular y por esta razon ponen todas sus actividades para que el público lo vea. Hacer eso facilita que *enemigos o gente con malas intencíones los encuentren. El interenet es algo muy divertido y puede ser algo positivo. Es necesario que la información revelada sea limitada y que solo información que *gente mala pueda ver sea escrita en estas paginas como Facebook. El articulo mensiona que solo los que cuidan su privacidad tienen derecho a quejarse cuando su información es revelada. Ojala y todo el mundo tenga ese derecho. [avanzado, 404, M]

\section{B. Abreviaturas de las glosas}

$\begin{array}{ll}\text { ATR } & \text { atributo } \\ \text { CC } & \begin{array}{l}\text { complemento circunstancial } \\ \text { determinante }\end{array} \\ \text { DET } & \text { nombre no contable } \\ \text { N.nc } & \text { nombre singular } \\ \text { N.S } & \text { nombre plural } \\ \text { N.pl } & \text { objeto directo } \\ \text { OD } & \text { predicado } \\ \text { PRED } & \text { sintagmas nominales } \\ \text { SSNN } & \text { verbo } \\ \text { V } & \text { verbo copulativo } \\ \text { VC } & \end{array}$

